\title{
SOCS1 restricts dendritic cells' ability to break self tolerance and induce antitumor immunity by regulating IL-12 production and signaling
}

\author{
Kevin Evel-Kabler,, ${ }^{1,2}$ Xiao-Tong Song,, ${ }^{1,3}$ Melissa Aldrich, ${ }^{1,2}$ Xue F. Huang, ${ }^{1,4}$ and Si-Yi Chen1,2,3
}

${ }^{1}$ Center for Cell and Gene Therapy, ${ }^{2}$ Department of Immunology, ${ }^{3}$ Department of Molecular and Human Genetics, and ${ }^{4}$ Department of Pediatrics, Baylor College of Medicine, Houston, Texas, USA.

\begin{abstract}
DC-based tumor vaccine research has largely focused on enhancing DC maturation/costimulation and antigen presentation in order to break tolerance against self tumor-associated antigens. DC immunization can activate autoreactive $T$ cells but rarely causes autoimmune pathologies, indicating that self tolerance at the host level is still maintained in the vaccinated hosts. This study in mice reveals a novel regulatory mechanism for the control of self tolerance at the host level by DCs through the restriction of positive cytokine feedback loops by cytokine signaling inhibitor SOCS1. The study further finds the requirement of persistent antigen presentation by DCs for inducing pathological autoimmune responses against normal tissues and tumor, which can be achieved by silencing SOCS1 to unleash the unbridled signaling of IL-12 and the downstream cytokine cascade. However, the use of higher-affinity self peptides, enhancement of DC maturation, and persistent stimulation with cytokines or TLR agonists fail to break tolerance and induce pathological antitumor immunity. Thus, this study indicates the necessity of inhibiting SOCS1, an antigen presentation attenuator, to break self tolerance and induce effective antitumor responses.
\end{abstract}

\section{Introduction}

The mechanisms utilized by professional APCs to sense microbes and initiate immune responses has been well studied (1-3), largely because of the critical role of APCs such as DCs in initiating and regulating immune responses and their therapeutic potential (4-6). When DCs encounter proinflammatory stimuli, such as microbial products, the maturation process is initiated and results in proinflammatory cytokine secretion and upregulation of MHC molecules and costimulatory molecules $(4,5)$. Following maturation and homing to LNs, DCs establish contact with T cells by forming an immunological synapse, where the TCR/MHC interaction and costimulatory molecules congregate in a central area surrounded by adhesion molecules (7). Once activated, $\mathrm{CD}^{+} \mathrm{CTLs}$ can proliferate for several generations and acquire lytic function $(8,9)$. It has therefore been proposed that the level and duration of peptide-MHC complexes (signal 1 ) and costimulatory molecules (signal 2) provided by DCs determines the magnitude and fate of an antigen-specific $\mathrm{T}$ cell response $(10,11)$. In addition, cytokines have been implied as a third signal for the activation of T cells by DCs $(12,13)$.

Little is known about the negative regulatory mechanisms in DCs used to control the magnitude and overactivation of T cell responses, in contrast to the extensive studies on DC activation. Several studies demonstrate that SOCS1 is a negative regulator of LPSinduced macrophage activation and plays an essential role in suppressing systemic autoimmunity mediated by DCs (14-16). SOCS1 is an inducible negative feedback inhibitor of the JAK/STAT signal

Nonstandard abbreviations used: ELISPOT, enzyme-linked immunospot; TAA, tumor-associated antigen; TRP2, tyrosinase-related protein 2.

Conflict of interest: The authors have declared that no conflict of interest exists.

Citation for this article: J. Clin. Invest. 116:90-100 (2006).

doi:10.1172/JCI26169. pathway (17). Homozygous genetic KO of SOCS1 in mice results in neonatal lethality due to uncontrolled IFN- $\gamma$ signaling $(18,19)$. SOCS1-KO DCs are hyperactivated and induce aberrant expansion of B and T cells. Recently we found that SOCS1-silenced DCs were able to induce an enhanced antigen-specific CTL response and antitumor activity (20). We have identified SOCS1 as an important negative regulator of antigen presentation by DCs and demonstrated that silencing of SOCS1 enhances antigen presentation by DCs and antigen-specific antitumor immunity (20). In agreement, Hanada et al. reported that immunization with SOCS1-KO DCs induced a hyper-Th1-type immune response and antitumor activities (21).

Immunologic peripheral tolerance to self antigen reflects the inability of autoreactive $T$ cells that escape censoring in the thymus to cause autoimmune pathologies. The essential requirement for an effective tumor vaccine is its ability to break self tolerance and induce pathological autoimmune responses against tumors and normal nonessential tissues that express self tumor-associated antigens (self TAAs) (22). DC vaccines have been viewed as 1 of the most promising strategies for tumor vaccination $(4,6,23,24)$. A puzzling paradox is that mature DC immunization can effectively break self tolerance at the cellular level, i.e., activate self antigen-specific CTLs, but rarely causes autoimmune pathologies against normal tissues and tumors (25), suggesting that self tolerance at the host level is still maintained in a host's natural immunosuppressive environment, sustained by various mechanisms such as regulatory $\mathrm{T}$ cells and exaggerated by the tumor-derived factors $(26,27)$, impeding the efficacy and usefulness of DC-based tumor vaccination.

The aims of this study were to investigate the underlying regulatory mechanisms in DCs used to control the magnitude and overactivation of autoreactive CTL responses and to define the requirements for DCs to induce TAA-specific, pathological autoimmune antitumor responses. We investigate autoimmune CTL responses 


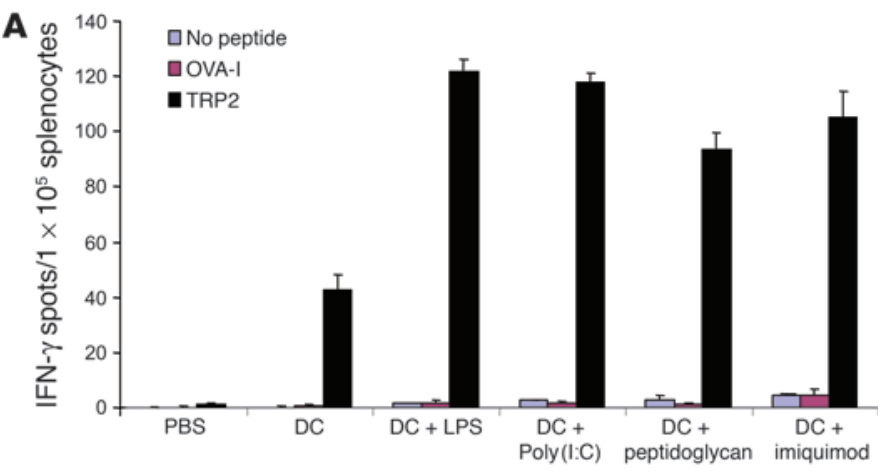

B

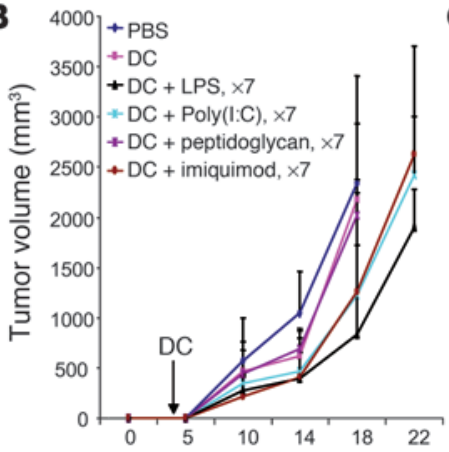

Days after tumor injection

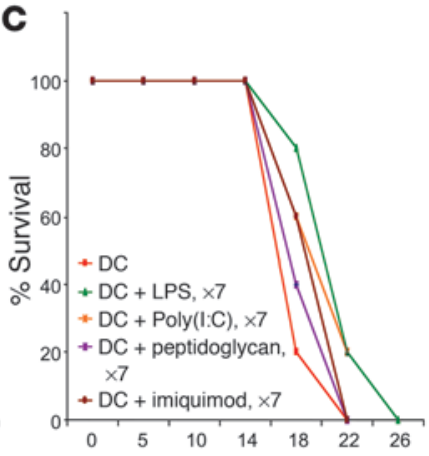

Days after tumor inoculation

\section{Figure 1}

Persistent TLR stimulation of DC immunization failed to induce pathological autoimmune response and antitumor immunity. (A) TRP2-specific CTL responses induced by persistent in vivo TLR stimulation and DC immunization. C57BL/6 mice were immunized with TRP2-pulsed $(100 \mu \mathrm{g} / \mathrm{ml})$ and LPS-matured $(100 \mathrm{ng} / \mathrm{ml})$ DCs and then stimulated with various TLR agonists daily for 7 consecutive days. Splenocytes pooled from immunized mice (2-3 mice) were subjected to IFN- $\gamma$ ELISPOT assays. An irrelevant peptide (OVA-I peptide) was used as a negative control. (B and C) Inability to control preestablished B16 tumors by persistent in vivo TLR stimulation and DC immunization. Groups of mice were inoculated s.c. with B16 tumor cells $\left(2.5 \times 10^{5}\right)$ and 3 days later were immunized via the rear foot pad with $1.5 \times 10^{6}$ TRP2 peptide-pulsed $(100 \mu \mathrm{g} / \mathrm{ml})$ DCs with ex vivo LPS maturation. After DC transfer, in vivo TLR agonists were administered i.p. daily for 7 days. Tumor growth and percent survival ( $n=5-7$ mice/group) curves represent 1 of 3 independent experiments. When their tumor volume reached approximately $2,000 \mathrm{~mm}^{3}$ in size, mice were euthanized and recorded as dead.

lation after DC transfer, the development of autoimmune vitiligo, a self-reactive immune response targeted against TRP2 in host melanocytes that is characterized by the development of coat lightening, depigmentation, and/or hair loss, was absent from all immunized mice. Immunization with TRP2-pulsed, matured DCs, followed by in vivo stimulation with various TLR agonists, failed to effectively control tumor progression (Figure $1, \mathrm{~B}$ and $\mathrm{C}$ ). These results indicate that this immunization protocol of mature DC transfer plus persistent in vivo TLR signaling can effectively break self tolerance at the cellular level, i.e. activate self antigen-specific CTLs, but not at the host level, as manifested by the lack of autoimmune pathologies against self antigen-expressing normal tissues and tumor.

Silencing of a signaling inhibitor allows DCs to break self tolerance and induce autoimmune pathologies. To investigate possible molecular restrictions in DCs to prevent autoimmune pathologies, we tested the effect of silencing SOCS1 in DCs on autoimmune responses based upon our previous findings (20). A lentiviral vector, LV-SOCS1siRNA (eYFP), with the ability to specifically downregulate approximately $90 \%$ of SOCS1 mRNA in BM-derived DCs and a control vector, LV-GFP-siRNA (eYFP), were generated as described previously (20). In agreement, the SOCS1 protein expression in the transduced LV-SOCS1-siRNA DCs was significantly reduced, as demonstrated by Western blotting (Supplemental Figure 1; supplemental material available online with this article; doi:10.1172/JCI26169DS1). TRP2pulsed, transduced DCs were transferred into WT C57BL/6 mice. The mice were then stimulated in vivo with or without a low dose of LPS, and CTL responses were measured. As the number of in vivo LPS stimulations was increased, the amount of TRP2-specific $\mathrm{CD}^{+}$ T cells also increased in SOCS1-siRNA DC mice (Figure 2A). In contrast, the amount of TRP2-specific $\mathrm{CD}^{+} \mathrm{T}$ cells was only marginally increased in GFP-siRNA DC mice, regardless of the number of LPS stimulations, and was consistently lower than that in SOCS1-siRNA DC mice (Figure 2A, left panels). IFN- $\gamma$ ELISPOT and CTL assays showed similar results (data not shown). The development of autoimmune vitiligo was apparent at 2-3 months after immunization in many of the SOCS1-siRNA DC mice injected with LPS (Figure 2B). In contrast, autoimmune vitiligo was not observed in any of GFPsiRNA DC mice, even with repeated in vivo LPS administrations. Although the mechanisms for the inability of GFP-siRNA DCs to 

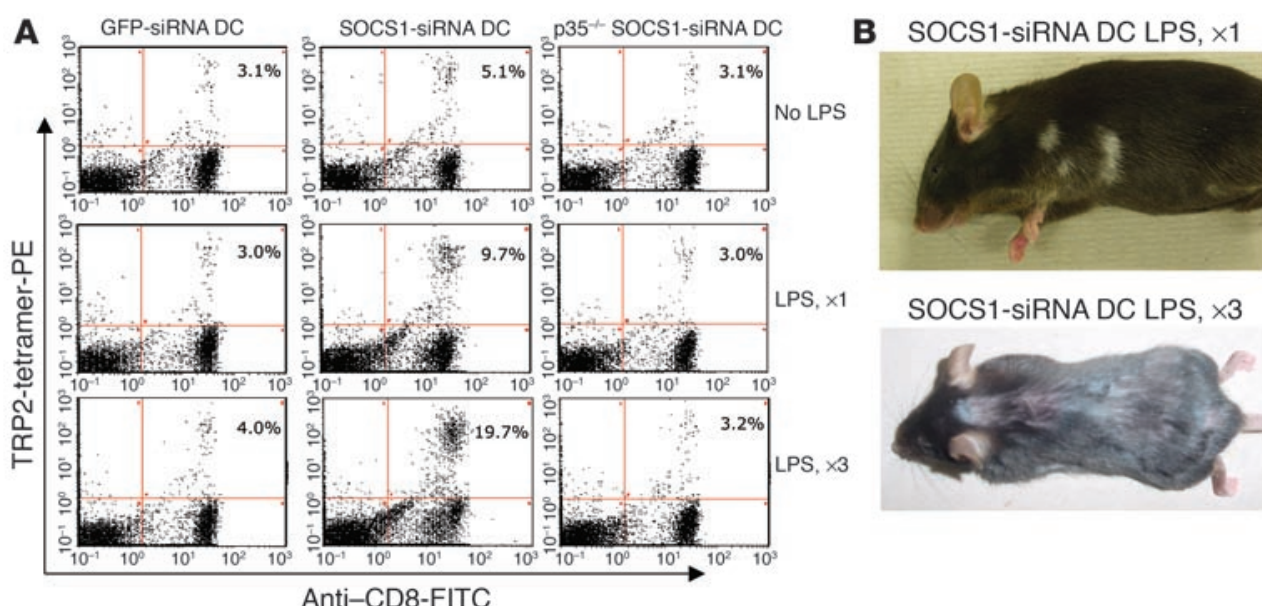

SOCS1-siRNA DC LPS, $\times 3$

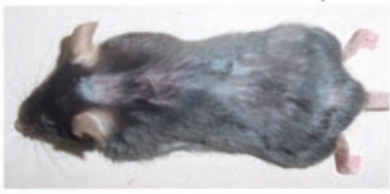

Anti-CD8-FITC

C

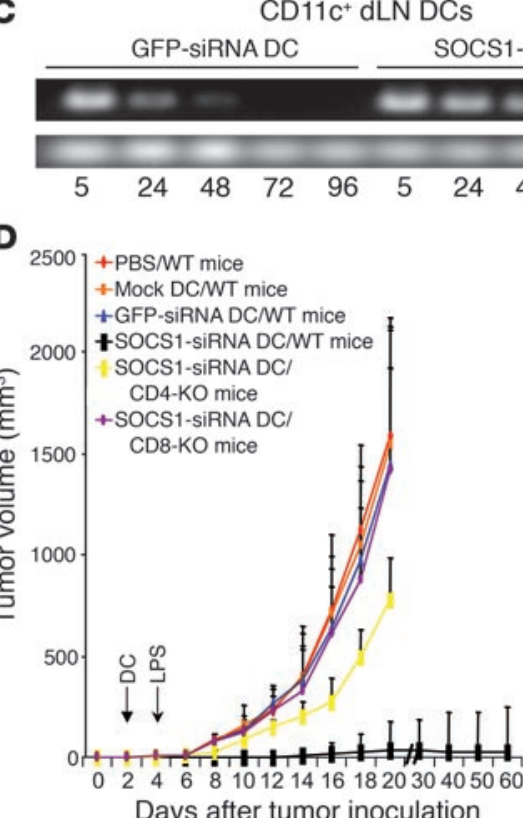

Days after tumor inoculation

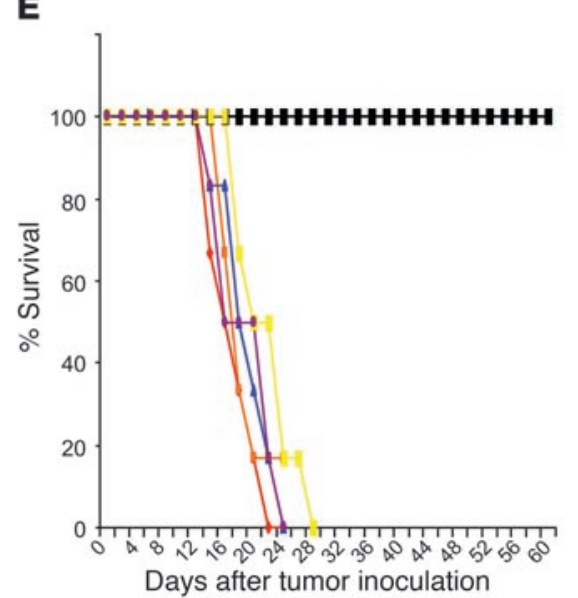

Figure 2

SOCS1-silenced DCs induced pathological autoimmune responses. (A) TRP2-specific CTL responses induced by SOCS1-silenced DCs. Mice were immunized with TRP2-pulsed $(50 \mu \mathrm{g} / \mathrm{ml})$, LV-transduced WT, or IL-12p35-KO DCs with LPS-induced maturation $(100 \mathrm{ng} / \mathrm{ml})$ ex vivo. The mice were then stimulated 0,1 (day 1), or 3 times (days 1, 4, and 7) i.p. with LPS (30 $\mu \mathrm{g} /$ mouse/injection). Percentages of TRP2tetramer-PE-positive T cells in the gated $C D 8^{+} \mathrm{T}$ cells of splenocytes in mice 2 weeks after immunization are shown from 1 of 3 independent experiments. $P<0.01$, GFP-siRNA DCs versus SOCS1-siRNA DCs. (B) Representative autoimmune vitiligo of mice 3 months after immunization with TRP2-pulsed LVSOCS1-siRNA DCs, followed by LPS stimulation once or 3 times. (C) Duration of DCs in draining LNs. Groups of mice were immunized with LV-transduced DCs, and CD11 $\mathrm{c}^{+} \mathrm{DC}$ s isolated from the draining LNs (dLN) of the mice at different times were used for RT-PCR analysis of the transgene eYFP marker mRNA. GAPDH was used as internal control. Experiments were repeated twice with similar results. (D and E) Inhibition of preestablished B16 tumors by SOCS1-siRNA DCs. WT, CD4-KO, or CD8-KO C57BL/6 mice were inoculated s.c. with B16 tumor cells $\left(2.5 \times 10^{5}\right)$ and 3 days later were immunized via the rear foot pad with $1.5 \times 10^{6}$ TRP2 peptide-pulsed $(50 \mu \mathrm{g} / \mathrm{ml})$, LV-transduced DCs with ex vivo LPS maturation (100 ng/ml). One day after DC transfer, in vivo LPS was administered i.p. $(30 \mu \mathrm{g} / \mathrm{mouse}) 1$ time. Tumor growth ( $n=6$ mice/group) and percent survival curves represent 1 of 3 independent experiments. $P<0.01$, GFP-siRNA DCs compared with SOCS1-siRNA DCs.

induce stronger antitumor immunity in response to repeated LPS stimulation are still not clear, SOCS1-restricted JAK/STAT signaling, endotoxin tolerance, and/or other mechanisms may contribute to this outcome. Interestingly, we observed that the duration of the presence of SOCS1-siRNA DCs in draining LNs of immunized mice was increased, compared with that of GFP-siRNA DCs (Figure $2 \mathrm{C})$, suggesting that the enhanced response of SOCS1-siRNA DCs to repeated LPS stimulation is, in part, due to the prolonged duration of these DCs in draining LNs. Taken together, these results suggest a critical role of SOCS1 in DCs for maintaining tolerance to self antigens at the host level and a necessity of silencing the signaling inhibitor to induce pathological autoimmune responses by DC immunization.

SOCS1-silenced DCs induce effective antitumor immunity capable of controlling preestablished B16 tumors when costimulated with a TLR agonist. We (20) and Hanada et al. (21) have independently shown that SOCS1-siRNA DC or SOCS1-KO DC vaccination of weakly immunogenic $\left(\right.$ TRP2 $\left.2^{+}\right)$B16 tumor-bearing mice results in a reduction in tumor growth and an increase in survival; however, tumor growth is not fully inhibited, and mice eventually succumb to tumor burden. Therefore, we tested whether SOCS1-silenced DCs more potently induce autoreactive CTL responses capable of controlling the growth of preestablished B16 tumors when boosted in vivo with a TLR agonist such as LPS, a potent inducer of proinflammatory cytokines (1-3). The addition of in vivo stimulation with LPS significantly enhanced the ability to inhibit B16 tumors and promote mouse survival by immunization with SOCS1-siRNA but not GFP-siRNA DCs (Figures 2, $\mathrm{D}$ and $\mathrm{E})$. The enhanced antitumor activity was correlated with potent TRP2-specific CTL activities observed in SOCS1-siRNA DC mice (data not shown). By transferring SOCS1-siRNA DCs into CD4- and CD8-KO mice, it was further demonstrated that the antitumor response required both $\mathrm{CD}^{+}$and $\mathrm{CD} 4^{+}$cells, although a weak antitumor response was observed in CD4-KO mice (Figures 2, D and E). No apparent toxicity, other than autoimmune vitiligo, was observed in the 


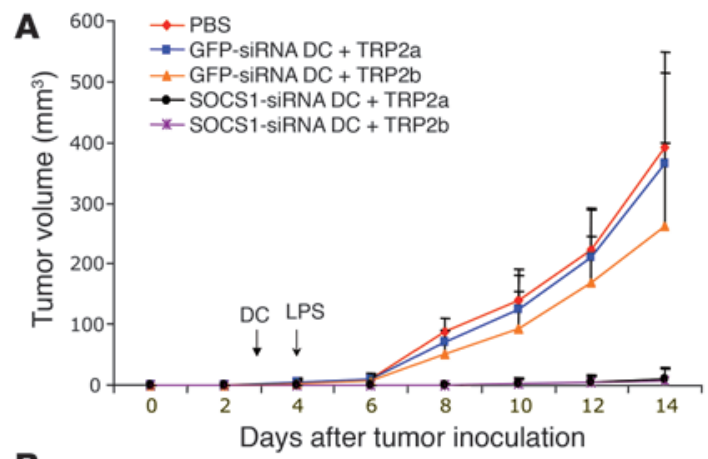

B

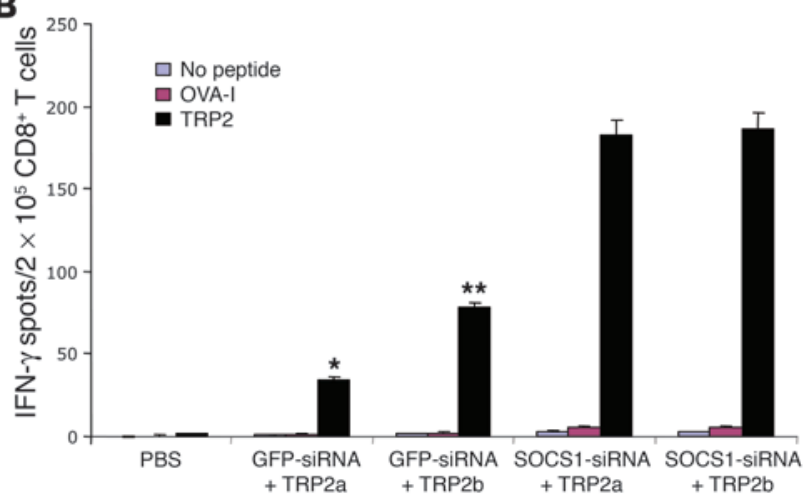

Figure 3

Effects of SOCS1 silencing on antigen presentation by DCs. (A) Inhibition of preestablished B16 tumor by SOCS1-siRNA DC immunization with TRP2 peptides of different affinities. WT C57BL/6 mice were inoculated s.c. with B16 tumor cells $\left(2.5 \times 10^{5}\right)$ and 3 days later were immunized with $1.5 \times 10^{6}$ TRP2 peptide-pulsed $(50 \mu \mathrm{g} / \mathrm{ml}$; TRP2a or TRP2b), transduced DCs with ex vivo LPS maturation $(100 \mathrm{ng} / \mathrm{ml})$. One day after DC transfer, in vivo LPS was administered i.p. (30 $\mu \mathrm{g} /$ mouse) 1 time. Tumor growth curves ( $n=6$ mice/group) represent 1 of 3 independent experiments. $P<0.01$, GFP-siRNA DC compared with SOCS1-siRNA DCs. (B). CD8+ T cell responses induced by SOCS1siRNA DCs with different TRP2 peptides. CD8 ${ }^{+} \mathrm{T}$ cells isolated from the pooled splenocytes of immunized mice (2-3 mice) were subjected to IFN- $\gamma$ ELISPOT assays stimulated with TRP2a or TRP2b peptide $(10 \mu \mathrm{g} / \mathrm{ml})$. An irrelevant peptide derived from ovalbumin was used as a negative control. ${ }^{*} P<0.01$ versus SOCS1-siRNA DC plus TRP2a; ${ }^{\star \star} P<0.01$ versus SOCS1-siRNA DC plus TRP2a.

TRP2-pulsed SOCS1-siRNA DC mice coinjected with LPS more than 6 months after immunization. Histological analysis of major organs and tissues of the immunized mice revealed no pathologic inflammation (data not shown). Collectively, these results suggest that SOCS1-restricted signaling of TLR agonist-induced cytokines in DCs may control their ability to break tolerance at the host level and induce effective antitumor immunity.

Role of SOCS1-restricted cytokine (IL-12) production by DCs in breaking self tolerance at the host level. We next investigated possible mechanisms used by SOCS1 in DCs to regulate antigen presentation by examining its influence on 3 major signals provided to T cells: antigenic peptide/MHC presentation (signal 1), costimulatory molecule expression (signal 2), and/or cytokine production (signal 3). By flow cytometric assays, we consistently found that there were only slightly altered surface levels of costimulatory/inhibitory molecules (B7.1, B7.2, OX40L, CD40, or PDL1) on SOCS1-siRNA DCs compared with those on GFP-siRNA DCs both before and after LPS-induced maturation (data not shown), in agreement with our previous observation (20). Comparable levels of MHC class I and II molecules (signal 1) were also detected on SOCS1-siRNA DCs and GFP-siRNA DCs (data not shown). We further investigated whether peptide immunogenicity (TCR affinity) can influence SOCS1-restricted CTL responses in vivo. Since a high-affinity form (TRP2b) and a low-affinity form (TRP2a) of the TRP2 CTL peptide were identified previously (28), we used these 2 TRP2 peptides to test whether the strength of signal 1 can influence the ability of transduced DCs to control B16 tumor growth. Figure 3A shows that GFP-siRNA DCs loaded with either the low- or high-affinity peptide were unable to induce B16 tumor regression with in vivo LPS stimulation, although GFP-siRNA DCs loaded with the TRP2b peptide showed a marginal effect on tumor growth. In contrast, both SOCS1-siRNA DC groups loaded with the low- or high-affinity TRP2 peptide effectively blocked tumor growth. We investigated TRP2-specific CTL activities in vaccinated mice with IFN- $\gamma$ ELISPOT. Figure 3B shows that GFP-siRNA DCs loaded with the high-affinity peptide induced stronger IFN- $\gamma$ responses than did GFP-siRNA DCs loaded with the low-affinity peptide. However, both SOCS1-siRNA DCs loaded with low- or high-affinity peptide induced much stronger IFN- $\gamma$ responses than GFP-siRNA DCs $(P<0.01)$. These results show that SOCS1 in DCs does not have a significant impact on the expression of costimulatory/inhibitory molecules or MHC class I and II molecules on DCs; and SOCS1-restricted signaling in DCs plays a more dominant role than self peptide affinity in inducing CTL responses and antitumor immunity.

The above observations and known SOCS1 functions as an inhibitor of JAK/STAT signaling (17) imply a critical role of SOCS1-restricted cytokine signaling and production in controlling $\mathrm{T}$ cell responses. We initially tested the importance of several candidate cytokines known to influence CTL activation by using DCs derived from the BM of different $\mathrm{KO}$ mice. LV-transduced DCs derived from IL-12p35-KO mice (p35-/- SOCS1-siRNA DCs) were no longer able to inhibit the growth of preestablished B16 tumors (Figures 4, A and B). IFN- $\gamma$ ELISPOT and CTL assays showed that p35 ${ }^{-/}$SOCS1-siRNA DCs had a substantially reduced ability to induce TRP2-specific CTL responses compared with WT SOCS1siRNA DCs (Figure 4C and Supplemental Figure 2). In addition, in vivo stimulation with LPS failed to boost CTL responses induced by $\mathrm{p} 35^{-/-}$SOCS1-siRNA DCs as measured by TRP2-tetramer analysis (Figure 2A), and autoimmune vitiligo did not develop in these mice (data not shown). In contrast to the essential role of IL-12 produced by antigen-presenting DCs, antitumor CTL responses were still effectively induced in IL-12p35-KO mice immunized with WT SOCS1-siRNA DCs, suggesting that IL-12 produced by host cells is not required for the induction of CTL responses (Figure 4, A-C, and Supplemental Figure 2). These results suggest that IL-12 production by SOCS1-silenced, antigen-presenting DCs is required for inducing a potent TRP2-specific CTL response and breaking self tolerance at the host level.

Prolonged and enhanced production of IL-12 by SOCS1-silenced DCs versus transient and low production by WT DCs. DCs produce significant amounts of heterodimeric IL-12 in response to microbial products and/or CD40 ligand. However, the duration of IL-12 production by stimulated DCs is tightly restricted to a short time period (8-16 hours) (31). We determined the effect of SOCS1 on the concentration and duration of IL-12 produced by DCs following in vitro stimulation. To test this we stimulated SOCS1-silenced DCs or control DCs with LPS alone or in combination with stimula- 

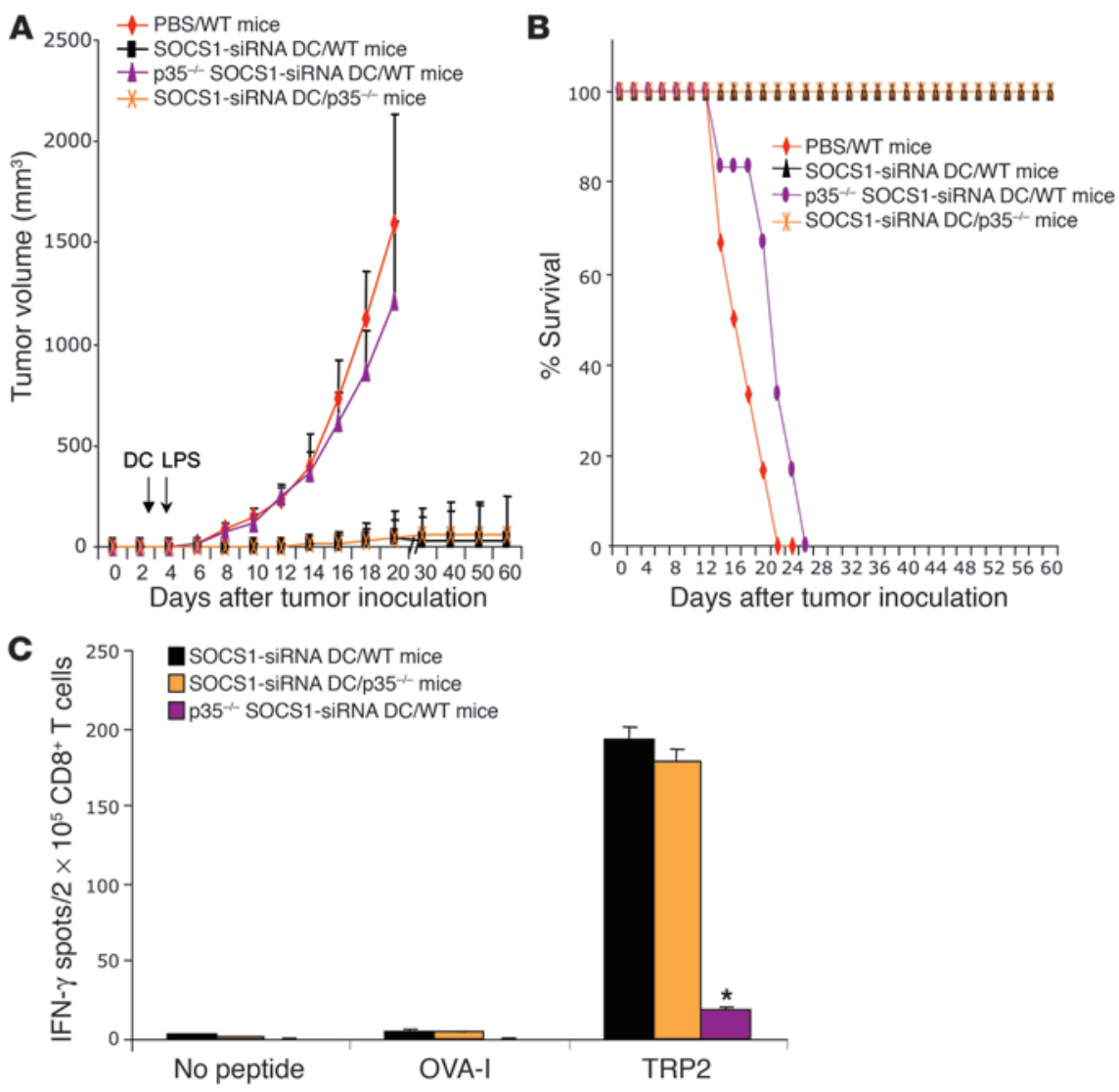

tory anti-CD40 antibody and then measured the production of IL-12. We observed a small but significant $(P<0.05)$ increase in the production of IL-12p40 by SOCS1-silenced DCs derived from BM culture with GM-CSF and IL-4, compared with control DCs (Figure 5A), which is consistent with a previous observation (32). Strikingly, the expression of IL-12p70 by SOCS1-siRNA DCs was substantially increased after stimulation compared with that by control DCs (Figure 5B), which is consistent with 2 recent reports that SOCS1-KO macrophages produced excessive amounts of IL-12 and other cytokines in response to stimuli and that enhanced levels of IL-12 and other cytokines were found in sera of conditional SOCS1-KO mice $(33,34)$. Interestingly, BM DCs after culture with only GM-CSF produced less IL-12 (Figure 5A), likely due to the previously described stimulatory effects of IL-4 on IL-12 production by DCs (35). The marked difference in IL-12p70 compared with IL-12p40 secretion is likely due to the upregulation of IL-12p35 expression, which has previously been shown to act as the ratelimiting step for IL-12 heterodimer secretion (36). Indeed, higher levels of IL-12p35 mRNA were also detected in SOCS1-siRNA DCs, compared with control GFP-siRNA DCs (Supplemental Figure 3). We also observed that the levels of the IFN-inducible factors ICSBP and IRF-1 mRNA (37) appeared enhanced in SOCS1siRNA DCs, while the transcription factors C/EBP- $\beta$ and c-Rel remained largely unchanged compared with control DCs (Supplemental Figure 3). Consistent with the in vitro data, the expression levels of IL-12p35 as well as other proinflammatory cytokines, IFN- $\gamma$ and IL-2, were elevated in DCs isolated from the draining LNs of mice receiving SOCS1-siRNA DCs and in vivo LPS stimulation (Supplemental Figure 4). Finally, we found that SOCS1silenced DCs were able to continuously produce high levels of

\section{Figure 4}

The role of IL-12 produced by SOCS1silenced DCs in breaking self tolerance and inducing antitumor immunity. (A and B) Inability to inhibit preestablished B16 tumor by IL-12-deficient SOCS1-siRNA DCs. WT or IL-12p35-KO mice were inoculated s.c. with B16 tumor cells $\left(2.5 \times 10^{5}\right)$ and 3 days later were immunized with $1.5 \times 10^{6}$ TRP2-pulsed, transduced WT, or IL-12p35-KO DCs with ex vivo LPS maturation. Tumor growth $(\mathbf{A})$ and percent survival (B) ( $n=6 \mathrm{mice} /$ group) curves represent 1 of 2 independent experiments. $P<0.01$, GFP-siRNA DCs compared with SOCS1-siRNA DCs. (C) CD8+ T cell responses induced by IL-12-deficient SOCS1-siRNA DCs. CD8+ $\mathrm{T}$ cells isolated from the pooled splenocytes of immunized WT or IL-12p35$\mathrm{KO}$ mice were subjected to IFN- $\gamma$ ELISPOT assays. An irrelevant peptide (OVA-I peptide) was used as a negative control. ${ }^{*} P<0.01$ versus SOCS1-siRNA DCs.

IL-12p70 for at least 72 hours when stimulated with LPS/anti-CD40 mAb, while control GFP-siRNA DCs produced only low levels of IL-12p70 over the same culture period (Figure 5C). These results indicate that SOCS1 silencing allows DCs to continuously produce increased levels of IL-12p70 in response to inflammatory stimulation.

Increased and continuous production of IL-12 by WT DCs is not sufficient to break self tolerance at the host level. To investigate whether overproduction of IL-12 alone may account for the ability of SOCS1-silenced DCs to break self tolerance at the host level, we compared the ability of WT DCs transfected with recombinant adenovirus constitutively expressing biologically active mouse IL-12 cytokine (Ad-IL-12) and SOCS1-silenced DCs to induce antitumor CTL responses. DCs transfected with Ad-IL-12 at an MOI of 300 produced a high level of IL-12p70, comparable to the IL-12p70 level produced by SOCS1-siRNA DCs after stimulation with LPS. Unexpectedly, immunization with mature TRP2pulsed DCs transfected with different MOIs of Ad-IL-12 failed to control the growth of preestablished B16 tumors (Figure 6A). In agreement, in mice immunized with Ad-IL-12 DCs (300 or 1,000 MOI), only modest increases in CTL responses were observed (Figure 6, B and C). Furthermore, we found that in vivo stimulation with a low dose of IL-12 did not significantly enhance CTL responses and antitumor activities in GFP-siRNA DC mice but significantly enhanced the antitumor CTL responses in SOCS1siRNA DC mice (Figure 6, A-C), suggesting the enhanced sensitivity of SOCS1-silenced DCs to IL-12 stimulation. Importantly, vitiligo was not induced in mice immunized with Ad-IL-12 DCs or GFP-siRNA DCs with IL-12 coinjection, while it was observed in about $40 \%$ of TRP2-pulsed, SOCS1-siRNA DC mice coinjected with a low dose of IL-12 (Supplemental Figure 5). These results indicate that continuous overexpression of IL-12 by WT DCs is not sufficient to break self tolerance at the host level and that SOCS1-restricted signaling of IL-12 in antigen-presenting DCs and the concentration of IL-12 produced by DCs play a critical role in controlling CTL responses and tolerance. 

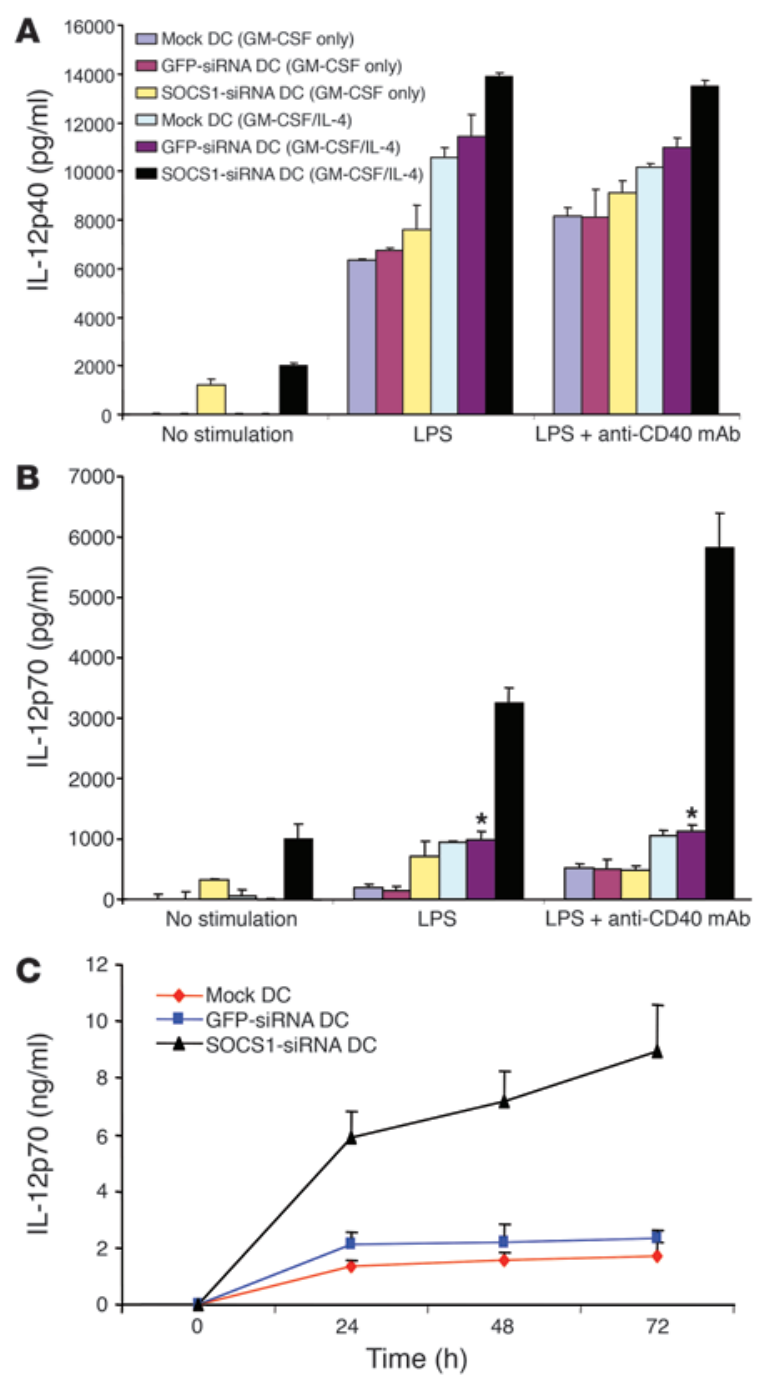

Figure 5

SOCS1 restricts the level and duration of IL-12 produced by DCs. (A and B) Levels of IL-12p40 (A) and IL-12p70 (B) secreted by DCs $\left(5 \times 10^{5}\right.$ cells $/ \mathrm{ml})$ in response to continuous stimulation with LPS $(100 \mathrm{ng} / \mathrm{ml})$ with or without plate-coated anti-CD40 mAb (1:1,000 dilution) for 24 hours from 1 of 3 independent experiments. All BM DCs were cultured with $\mathrm{mGM}-\mathrm{CSF}(20 \mathrm{ng} / \mathrm{ml})$ in the presence or absence of $\mathrm{mlL}-4(20 \mathrm{ng} / \mathrm{ml})$. ${ }^{*} P<0.01$ versus SOCS1-siRNA DCs. (C). Kinetics of IL-12p70 production by DCs $\left(5 \times 10^{5} \mathrm{cells} / \mathrm{ml}\right)$ in response to continuous stimulation with LPS $(100 \mathrm{ng} / \mathrm{ml})$ and anti-CD40 mAb for 0-72 hours from 1 of 3 independent experiments. All cells were cultured in the presence of both GM-CSF and IL-4. * $P<0.01$ GFP-siRNA DCs versus SOCS1-siRNA DCs.

Unbridled IL-12 signaling in DCs is required to break self tolerance at the host level. To test the role of IL-12 signaling in DCs for breaking self tolerance at the host level, we compared the CTL responses induced by WT DCs and DCs derived from IL-12 receptor-KO mice (IL-12R $\beta 2^{-/-}$). SOCS1-silenced DCs derived from IL-12R $\beta 2^{-/-}$SOCSsiRNA DCs exhibited a substantially reduced ability to induce TRP2specific CTLs compared with WT SOCS1-siRNA DCs (Figures 6C and 7B). Furthermore, p35-/- SOCS1-siRNA DCs and IL-12R $32^{-/-}$ SOCS1-siRNA DCs were unable to induce either tumor regression (Figure 7A) or autoimmune vitiligo (data not shown). We found that p35-/- SOCS1-siRNA DCs and IL-12R $32^{-/-}$SOCS1-siRNA DCs had a substantially reduced ability to induce TRP2-specific CTLs compared with WT SOCS1-siRNA DCs (Figure 7B). To further investigate the role of IL-12 signaling in DCs, we examined the ability of in vivo IL-12 stimulation to enhance CTLs in mice immunized with IL-12R $\beta 2^{-/-}$SOCS1-siRNA DCs. Figures 7, C and D, show that in vivo IL-12 stimulation of IL-12R $\beta 2^{-/-}$SOCS1-siRNA DC mice failed to enhance TRP-specific CTL responses. Collectively, these results indicate that SOCS1-restricted IL-12 signaling in antigen-presenting DCs plays a critical role in controlling antigen presentation and the unbridled signal transduction of IL-12 in antigen-presenting DCs is required to break self tolerance and induce pathological autoimmune responses.

SOCS1 restricts $I L-12$ and cytokine signaling cascade in DCs. Our data suggest that silencing SOCS1 may allow the establishment of an unbridled signaling cascade of IL-12 and IL-12-induced downstream cytokines in DCs. To further determine the ability of IL-12 to continuously signal in SOCS1-siRNA DCs, we examined the duration of IL-12 signaling in WT and SOCS1-silenced DCs by analyzing the phosphorylation kinetics of Stat 4 (phosphorylated Stat4 [pStat4]), which is primarily used by members of the IL-12 cytokine family (36). Figure 8A shows that Stat 4 was persistently phosphorylated for at least 72 hours in SOCS1-silenced DCs after LPS/anti-CD40 stimulation, whereas pStat4 was already at basal levels at 24 hours in GFP-siRNA DCs, in agreement with the continuous secretion of IL-12p70 by SOCS1-silenced DCs in response to LPS/anti-CD40 mAb stimulation shown in Figure 5C. Furthermore, Ad-IL-12-transfected DCs were found to only have low levels of pStat 4 at the indicated time points, suggesting that SOCS1 inhibits Stat 4 activation in Ad-IL-12 DCs that continuously produced and exposed to IL-12 (Supplemental Figure 6). We then examined whether IL-12 induces the enhanced production of downstream cytokines by SOCS1-silenced DCs. Figure 8B shows that IL-12 stimulation induced substantially higher levels of inflammatory cytokines such as IFN- $\gamma$ by mature SOCS1-siRNA DCs than by mature GFP-siRNA DCs, suggesting that SOCS1silenced DCs have an increased sensitivity to IL-12 stimulation. Figure 8C shows that GFP-siRNA DCs and mock-transduced DCs only transiently produced IL-12p70. In contrast, SOCS1-siRNA DCs persistently produced IL-12p70 after stimulation, at levels comparable to Ad-IL-12 DCs (MOI, 300). Importantly, this increased level of IL-12p70 secretion by SOCS1-siRNA DCs was dependent on the expression of the IL-12 receptor (Figure 8C), indicating that the marked increase in IL-12p70 secretion by SOCS1-silenced DCs is likely due to autocrine signaling by IL-12p70. IL-12 stimulation induces IFN- $\gamma$ production by DCs (38), and IFN- $\gamma$ was recently found to be hyper-produced by SOCS1-KO DCs in response to LPS (21). Therefore, we examined whether an unbridled cytokine network, not just IL-12, in SOCS1-silenced DCs is collectively responsible for inducing effective anti-tumor immunity. Using IFN- $\gamma-\mathrm{KO}$ DCs, we found that IFN- $\gamma$-KO SOCS1-siRNA DCs only induced weak CTL responses and did not control the growth of preestablished B16 tumors (Figure 7, A and B), suggesting an important role of IFN- $\gamma$ in inducing antitumor CTL responses by SOCS1siRNA DCs as well. Collectively, these data suggested that SOCS1 restricts cytokine signaling and only allows DCs to activate CTLs in a pulsing or transient fashion, thus limiting autoimmune pathologies. These data further suggest that SOCS1 silencing leads to the unbridled signaling of IL-12 and the downstream cytokine network in antigen-presenting DCs, resulting in the breaking of self tolerance at the host level and pathological autoimmune responses. 


\section{Discussion}

This study uncovers a novel regulatory mechanism for the control of pathological autoimmune responses by DCs through the restriction of cytokine signaling and the responsiveness to stimuli by SOCS1. We find that optimal immunization with matured WT DCs loaded with high-affinity self peptide (TRP2) plus persistent TLR signaling effectively activate self-reactive $\mathrm{T}$ cells but fail to break self tolerance at the host level. In contrast, SOCS1-silenced DCs effectively break tolerance at the host level and cause self antigen-specific, autoimmune pathologies against normal tissues and tumors. These results indicate that the requirement for breaking self tolerance at the cellular level (autoreactive CTL activation) and at the host level (autoimmune pathologies) by DCs is different and that the hardwired signaling inhibitor prevents DCs from overactivating autoreactive CTLs and causing autoimmune pathologies even when potent inflammatory stimuli are persistently present.

The subsequent mechanistic studies reveal that the inability of matured WT DCs plus persistent TLR signals to break self tolerance at the host level is likely due to the restriction of IL-12 and the downstream cytokine cascade signaling by SOCS1. This conclusion is supported by the following observations: (a) The lack of IL-12 production by SOCS1-silenced DCs (derived from IL-12p35-KO mice) abrogates their ability to break self tolerance at the host level; (b) The blockade of IL-12 signaling in SOCS1-silenced DCs (derived from IL-12R $\beta 2-\mathrm{KO}$ mice) also abrogates their ability to induce autoimmune pathologies and antitumor activities; (c) SOCS1-silenced DCs produce enhanced levels of IL-12p70 and downstream cytokines with a prolonged duration in response to stimulation; (d) In vivo administration of IL-12 as well as TLR agonists more effectively enhance CTL responses induced by SOCS1-silenced DCs than by WT DCs; and (e) WT DCs transduced with Ad-IL-12 to constitutively overexpress IL-12 are unable to induce autoimmune pathologies.

Maturation is known as the control point for DC transition from the immature, tolerogenic state to the activated, immunogenic state $(4,5)$. Our results demonstrate that proinflammatory cytokine signaling in mature DCs, restricted by SOCS1, critically regulates the magnitude of CTL responses. This indicates that CTL responses are controlled by DCs on at least 2 levels: the well-defined DC maturation required for the initiation of CTL responses and the SOCS1-restricted cytokine signaling of matured DCs for the control of the magnitude and overactivation of CTL responses uncovered in this study. Our results also imply dynamic interactions between DCs and their surrounding environment of various immune cells and stimuli, which collectively determines the magnitude of CTL responses. It has recently been demonstrated that mature DC lifespan is much longer than previously estimated, lasting $2-3$ weeks in vivo $(39,40)$, supporting the necessity of regulating antigen-presenting DCs after maturation.

This study establishes a new principle for breaking self tolerance and inducing pathological autoimmune responses, which is the first requirement for an effective tumor vaccine, by DCs via silencing a signaling inhibitor. The results of this study suggest the requirement of persistent antigen presentation by DCs for inducing autoimmune pathologies and effective antitumor immunity. Importantly, persistent antigen presentation by DCs cannot be achieved by persistent stimulation with proinflammatory stimuli or overproduction of a key proinflammatory cytokine (IL-12), probably because the hardwired signaling inhibitor prevents the establishment of an intricate autocrine/paracrine cytokine signaling network in DCs and permits DCs to stimulate CTLs only in a tran-
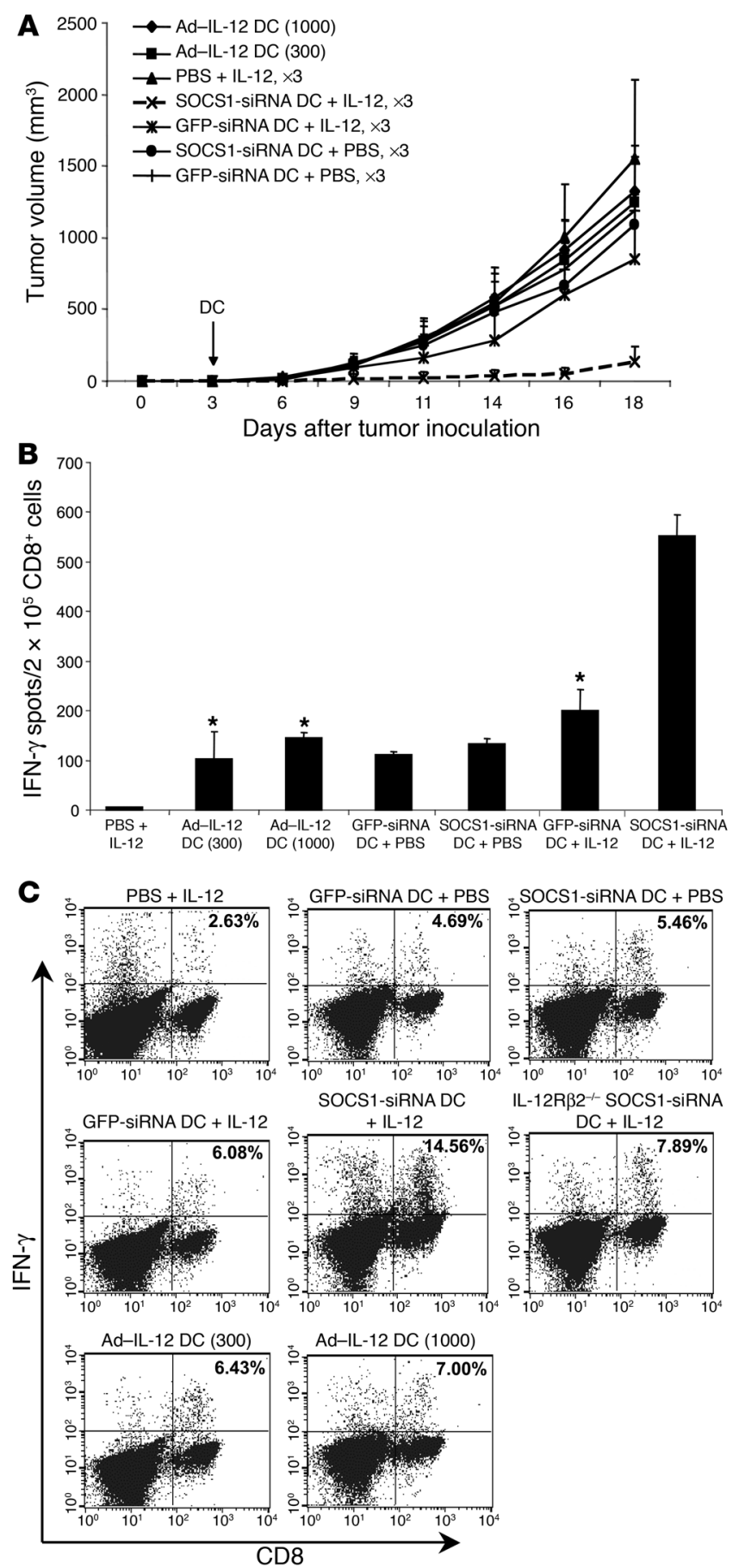

Figure 6

WT DCs constitutively expressing IL-12 were insufficient to overcome self tolerance at the host level. (A) Inability to inhibit preestablished B16 tumors by Ad-IL-12 DCs. BM DCs were transduced with Ad-IL-12 (MOls of 300 or 1,000 ) or LV-SOCS-siRNA or LV-GFP-siRNA (MOI of 5). WT mice were inoculated s.c. with B16 tumor cells $\left(2.5 \times 10^{5}\right)$ and 3 days later were immunized with $1.5 \times 10^{6}$ TRP2-pulsed DCs with ex vivo TNF- $\alpha$ maturation $(50 \mathrm{ng} / \mathrm{ml})$. After transfer of DCs, IL-12 ( $1 \mu \mathrm{g} /$ mouse) was administered i.p. 3 times on days 1,3 , and 5 . Tumor growth curves ( $n=6$ mice/group) represent 1 of 3 independent experiments. (B and C). Antigen-specific CD8 ${ }^{+} \mathrm{T}$ cell responses. Splenocytes or $\mathrm{CD}^{+} \mathrm{T}$ cells isolated from the pooled splenocytes of immunized mice were subjected to IFN- $\gamma$ ELISPOT assays (B) or intracellular IFN- $\gamma$ staining (C). ${ }^{*} P<0.01$ versus SOCS1-siRNA DCs plus IL-12. 
A

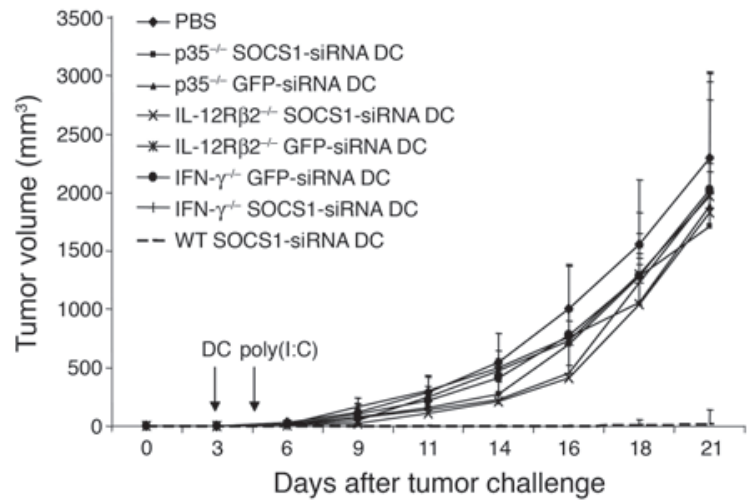

B
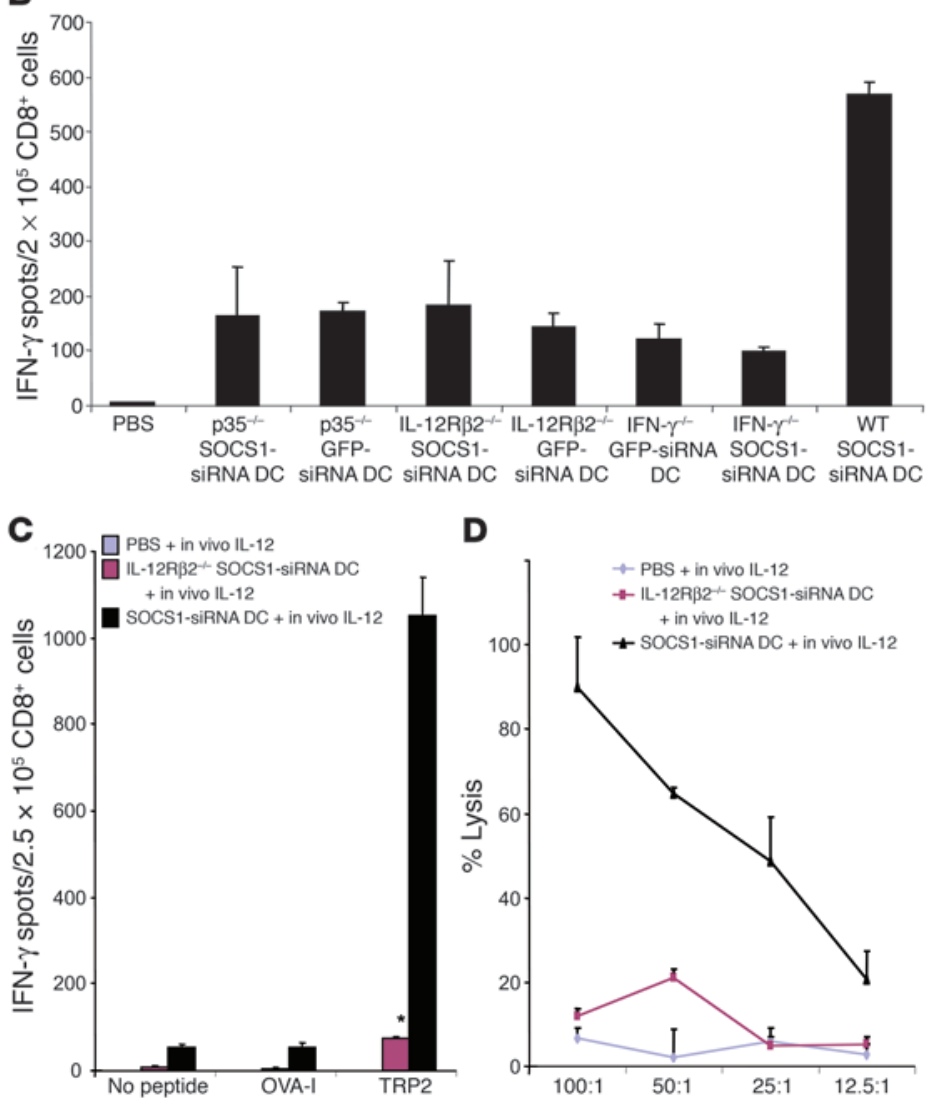

D

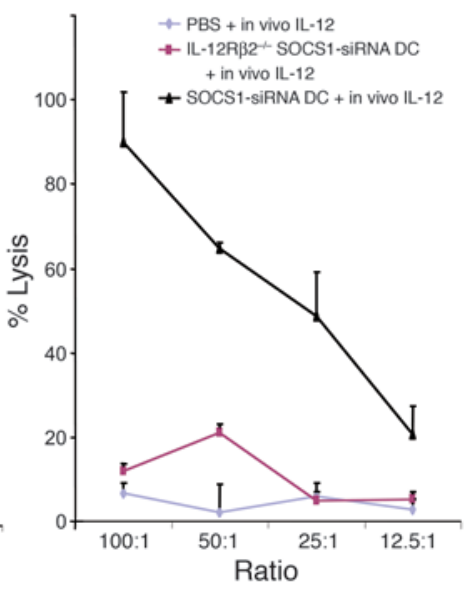

Figure 7

Persistent cytokine signaling in DCs is required to induce pathological autoimmune pathologies. (A) Inability to inhibit preestablished B16 tumors by IL-12p35---, IL-12R $32^{-1-}$, or IFN- $\gamma^{-1-}$ DCs. BM DCs derived from either WT, IL-12p35 $5^{--}$, IFN- $\gamma^{-1-}$, or IL-12R $32^{-/-}$mice were transduced with LV-SOCS1-siRNA or LV-GFP-siRNA. WT mice were inoculated s.c. with B16 tumor cells $\left(2.5 \times 10^{5}\right)$ and 3 days later were immunized with $1.5 \times 10^{6} \mathrm{TRP} 2$-pulsed, matured DCs, followed by poly(I:C) stimulation i.p. on days 1,3 , and 5 . Tumor growth curves ( $n=6$ mice/group) represent 1 of 3 independent experiments. (B) Reduced potency to induce CTL responses by SOCS1-silenced, IL-12R $\beta 2^{-/-}$DCs or IFN- $\gamma^{-1-}$ DCs. CD8 ${ }^{+} \mathrm{T}$ cells isolated from the pooled splenocytes of mice immunized with DCs derived from WT, IFN- $\gamma^{-1-}$, IL-12p35 $5^{-/}$, or IL-12R $\beta 2^{-1-}$ mice, followed by poly(I:C) stimulation 3 times, were subjected to IFN- $\gamma$ ELISPOT assays. ${ }^{*} P<0.01$ versus SOCS1-siRNA DCs. (C and $\mathbf{D}$ ) In vivo IL-12 stimulation failed to enhance the potency of SOCS1silenced, IL-12R $\beta 2^{--}$DCs. Mice were immunized once with WT or IL-12R $\beta 2^{--}$DCs $\left(1.5 \times 10^{6} /\right.$ mouse $)$ pulsed with TRP2 and matured with TNF- $\alpha$, followed by IL-12 (i.p.) 3 times. Two weeks later, splenocytes or $\mathrm{CD}^{+} \mathrm{T}$ cells isolated from the pooled splenocytes were subjected to IFN- $\gamma$ ELISPOT (C) and CTL assays against B16 tumor cells (D). ${ }^{*} P<0.01$ versus SOCS1-siRNA DCs.

Various inhibitory mechanisms exist in T cells to prevent pathological autoimmunity $(26,43,44)$. Disabling of 1 of these inhibitory mechanisms, such as the blockade of CTLA 4 on T cells or depletion of $\mathrm{CD} 25^{+}$regulatory $\mathrm{T}$ cells, is sufficient to break self tolerance at the host level but causes unwanted nonspecific autoimmune pathologies (26, 45, 46). However, inhibition of a signaling inhibitor in antigenloaded DCs can induce a TAA-specific, pathological autoimmune response against tumor. The results of our study also suggest a new avenue to improve tumor immunotherapy in general by coadministration of an inhibitor of these signaling inhibitors and a cytokine or TLR agonist.

DCs activated by TLR signaling produce a large number of cytokines, including IL-12, TNF- $\alpha$, IL- 6 , IFN- $\alpha / \beta$, and IFN- $\gamma$, most of which are regulated by SOCS1 $(17,47)$. The importance of cytokines as a third signal for the activation or overactivation of T cells by DCs has been recognized $(12,13,48)$. Production and signaling of proinflammatory cytokines such as IL-12 by WT DCs after TLR signaling is transient (31). Recent studies also showed that autocrine and paracrine signaling of cytokines produced by DCs plays a role in determining the outcome of antigen presentation $(30,49)$. Here we find that sient or pulsing fashion, thus limiting pathological autoimmune responses. The requirement of silencing a signaling inhibitor in order to achieve persistent antigen presentation by DCs for inducing autoimmune pathologies is in concept consistent with earlier findings that vaccination with GM-CSF-producing B16 melanoma cells caused vitiligo only when coinjected with anti-CTLA4 blocking antibodies for inhibiting the negative regulator on T cells (28, 41). However, the requirement for breaking tolerance in transgenic mouse models seems less stringent $(29,42)$. For example, Yang et al. recently showed that persistent in vivo TLR stimulation of WT DCs could break self tolerance at the host level in a transgenic viral antigen model. However, the conclusion drawn from the transgenic mouse models $(29,42)$ is not supported by the fact that autoimmune diseases are rare even in individuals with chronic infection with pathogens producing various TLR ligands. unbridled IL-12 and the downstream cytokine signaling play a critical role in the breaking of tolerance and increased CTL responses. Interestingly, SOCS1 1 - inflammatory disease is primarily caused by unbridled IFN- $\gamma$ signaling (17), although IL-12 also plays a role (47), while LPS-induced toxicity in SOCS1 $1^{--}$mice is linked to unbridled IFN- $\alpha / \beta$ signaling (32). Hanada et al. recently reported that SOCS1KO DCs induced more effective antitumor CTL responses probably due to the enhanced expression of IFN- $\gamma(21)$. Apparently, other cytokines such as IFN- $\gamma$ (Figure 7), in addition to IL-12, also play a role in the increased CTL responses induced by SOCS1-silenced DCs. Thus, further studies are warranted to delineate the cellular and molecular details of this SOCS1-regulated, complicated autocrine and paracrine cytokine signaling network in controlling the ability of DCs to activate or overactivate autoreactive CTLs in a natural, suppressive environment. In summary, this study contributes 
A

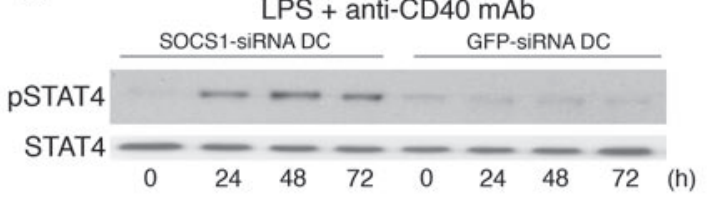

c

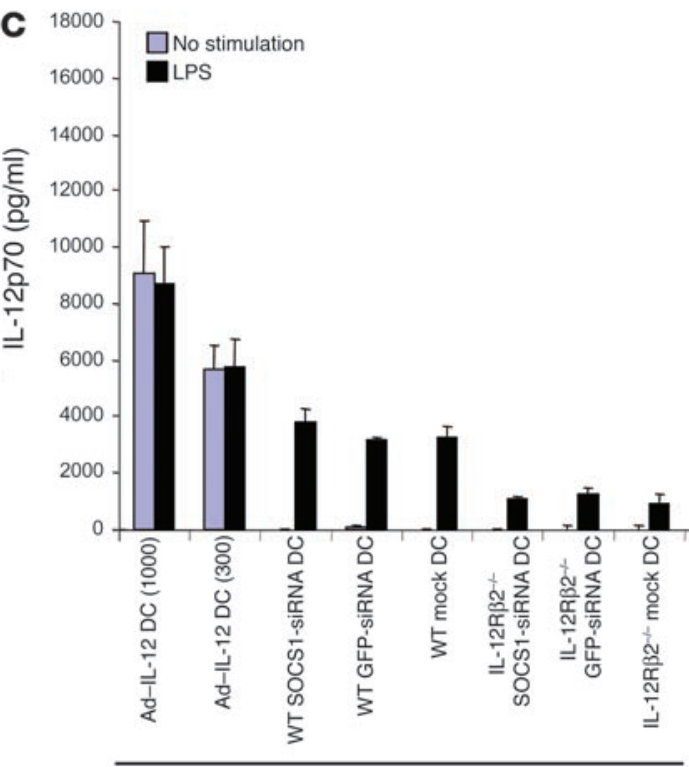

$6 \mathrm{~h}$
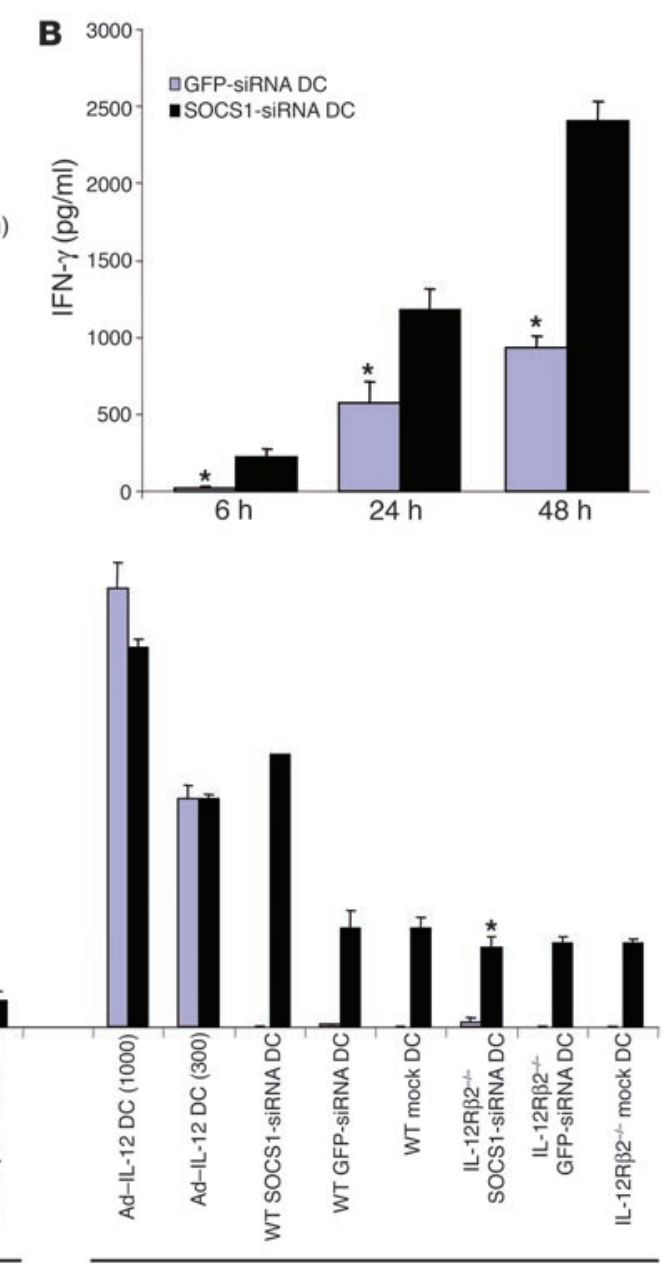

$24 \mathrm{~h}$

Figure 8

Enhanced signaling and sensitivity of SOCS1-silenced DCs to autocrine/paracrine IL-12 stimulation. (A) Western blotting of Stat4 and phosphorylated Stat4 (pStat4) in LV-transduced BM-DCs $\left(1 \times 10^{6}\right.$ cells $\left./ \mathrm{ml}\right)$ after stimulation with LPS and anti-CD40 mAb for 0, 24, 48 , or 72 hours. BM DCs were cultured in the presence of GM-CSF and IL-4. (B) IFN- $\gamma$ levels secreted by LV-transduced, TNF- $\alpha-$ matured WT DCs $\left(1 \times 10^{6}\right.$ cells $\left./ \mathrm{ml}\right) 6,24$, or 48 hours after stimulation with recombinant IL-12 $(20 \mathrm{ng} / \mathrm{ml})$ from 1 of 3 independent experiments. ${ }^{*} P<0.01$ versus SOCS1-siRNA DCs. (C) Levels of IL-12p70 secreted by transduced DCs $\left(1 \times 10^{6} \mathrm{cells} / \mathrm{ml}\right)$ derived from WT or IL-12R $\beta 2^{-/-}$mice at different times after stimulation with TLR agonist (LPS, $100 \mathrm{ng} / \mathrm{ml}$ ) from 1 of 3 independent experiments. All cells were cultured in the presence of GM-CSF and IL-4. ${ }^{\star} P<0.01$ versus WT SOCS1-siRNA DCs.

to the fundamental understanding of molecular and immunologic regulation of DCs and may lay a foundation for developing more effective tumor vaccines that not only activate autoreactive $\mathrm{T}$ cells, but also cause pathological autoimmune antitumor responses.

\section{Methods}

Mice. Approval for performing these mouse experiments was obtained from the institutional review board at Baylor College of Medicine. Four- to 6-week-old female C57BL/6, CD4-KO, CD8-KO, IL-12p35-KO, IFN- $\gamma-\mathrm{KO}$, and IL-12R $\beta 2-\mathrm{KO}$ mice were purchased from The Jackson Laboratory and maintained in a pathogen-free mouse facility at Baylor College of Medicine according to institutional guidelines.

Peptides. H2- $\mathrm{K}^{\mathrm{b}}$-restricted TRP2 peptides, TRP2a (VYDFFVWL) and TRP2b (SVYDFFVWL) (28), were used for this study. The control H2-K ${ }^{\mathrm{b}}-$ restricted peptide was OVA-I (SIINFEKL). All peptides were synthesized and purified by HPLC to greater than $95 \%$ purity by Genemed Synthesis Inc. All peptides were dissolved in DMSO before final dilution in endotoxin-free PBS.
Transduction of BM-derived DCs with lentiviral and adenoviral vectors. Recombinant lentiviral vectors (LV-SOCS1-siRNA and LV-GFP-siRNA) were produced and titrated as described previously (20). A recombinant adenovirus engineered to constitutively express biologically active IL-12 was purchased from InvivoGen and produced according to the manufacturer's instructions. Mouse BM-derived DCs were prepared as described previously (20). Briefly, mouse BM was flushed from the hind limbs, passed through a nylon mesh, and depleted of red cells with ammonium chloride. After extensive washing with RPMI-1640, cells were cultured with RPMI-1640 supplemented with $10 \%$ FBS, recombinant mouse GM-CSF/ml (20 ng/ml; PeproTech), and recombinant mouse IL-4 $(20 \mathrm{ng} / \mathrm{ml}$; PeproTech). On days 2 and 4 of culture, the supernatant was removed and replaced with fresh medium containing $\mathrm{mGM}-\mathrm{CSF}$ and $\mathrm{mIL}-4$. All cultures were incubated at $37^{\circ} \mathrm{C}$ in $5 \%$ humidified $\mathrm{CO}_{2}$. Nonadherent granulocytes were removed after 48 hours of culture, and fresh medium was added. After 7 days of culture, more than $80 \%$ of the cells expressed characteristic DC-specific markers as determined by FACS. Transductions of mouse BM-derived DCs (day 5-7 of culture) were performed on 
12-well plates with addition of $8 \mu \mathrm{g} / \mathrm{ml}$ Polybrene (Sigma-Aldrich) only for lentivirus-mediated transduction. DCs were washed and plated in 12-well plates at a concentration of $1 \times 10^{6}$ cells/well in $400 \mu \mathrm{l}$ of RPMI- 1640 . The cells were exposed to lentiviral or adenoviral vectors with different MOIs. After 8-12 hours of transduction, the cells were washed with PBS and further incubated in fresh tissue culture medium supplemented with GM-CSF and IL-4 as described previously $(20,50)$.

Cytokine ELISA analysis. Levels of various cytokines (IL-12p40, IL-12p70, IFN- $\gamma$ ) were quantitated using the supernatant of DC cultures using ELISA analysis (BD Biosciences) according to the manufacturer's instructions at the time points and with the stimulus as indicated in the figures.

RT-PCR. Total cellular RNA was isolated from DCs using RNeasy (QIAGEN) according to the manufacturer's instructions. The expression level of GAPDH was first evaluated as an internal control using serially diluted reverse-transcribed cDNA. The expression levels of the target mRNAs were then assessed using appropriate pairs of sense and antisense primers: GAPDH, 5'-ACCACAGTCCATGCCATCAC-3' and 5'-TCCACCACCCTGTTGCTGTA-3'; IL-12p35, 5'-AAATGAAGCTCTGCATCCTGC-3' and 5'-TCACCCTGTTGATGGTCACG-3' ;L-2, 5' -CTTCAAGCTCCACTTCAAGCT-3' and $5^{\prime}$-CCATCTCCTCAGAAAGTCCACC-3'; IFN- $\gamma, 5^{\prime}$-TCAAGTGGCATAGATGTGGAAGAA- $3^{\prime}$ and $5^{\prime}$-TGGCTCTGCAGGATTTTCATG-3'; eYFP, 5'-CACAAGTTCAGCGTGTCCGGC-3' and 5'-TCCAGCAGGACCATGTGATGC-3'; IRF-1, 5' -CCTGATGACCACAGCAGTTAC- $3^{\prime}$ and $5^{\prime}$-CTTCATCTCCGTGAAGACATG-3'; ICSBP, 5'-GATCAAGGAACCTTCTGTGG-3' and $5^{\prime}$-GAAGCTGATGACCATCTGGG-3'; c-Rel, 5'-TGGCTGACTGACTCACTGACTGACTGACTCGTGCCTTGC-3' and $5^{\prime}$-CCAACTAAATCATGAGGATGAGGCTTATATGGATCATTC-3'; C/EBP- $\beta, 5^{\prime}$-GCGGCGAGCGCAACAACATCT-3' and 5'-TGCTTGAACAAGTTCCGCAG-3'. For analysis of in vivo eYFP-expressing DC lifespan and IL-12p35, CD11 $\mathrm{c}^{+}$cells were first purified from the draining LNs using MACS (Miltenyi Biotec). The PCR products were separated by $1 \%$ agarose gel electrophoresis and visualized by ethidium bromide staining.

${ }^{51} \mathrm{Cr} C T$ L assays. $\mathrm{CD}^{+} \mathrm{CTL}$ responses were assessed with a standard chromium release assay, which measures the ability of in vitro-restimulated splenocytes to lyse target cells $(20,50)$. Splenocytes pooled from immunized mice were restimulated in vitro in RPMI-1640 containing H2-K $/$ TRP2 peptide for 4-6 days. TRP2 ${ }^{+}$target B16 cells, which express TRP2, and control EG.7 cells were labeled with sodium ${ }^{51} \mathrm{Cr}$ chromate solution for 90 minutes at $37^{\circ} \mathrm{C}$ with shaking. Different numbers of effector cells were incubated with a constant number of target cells $\left(5 \times 10^{4} /\right.$ well $)$ in 96 -well U-bottomed plates $(200$ $\mu \mathrm{l} /$ well) for 4 hours at $37^{\circ} \mathrm{C}$. The supernatants from triplicate cultures were collected. Percent lysis was calculated as (experimental release - spontaneous release) / (maximum release - spontaneous release) $\times 100$.

IFN- $\gamma$ ELISPOT assay. ELISPOT assays of isolated $\mathrm{CD}^{+} \mathrm{T}$ cells were performed as described in our previous studies (20). H2-Kb/TRP2 peptide was used for $\mathrm{CD}^{+} \mathrm{T}$ cell stimulation. Irrelevant peptide from OVA was also used as a negative control. Splenocytes or $\mathrm{CD}^{+}$cells were isolated from splenocytes using MACS CD8 (Ly-2) MicroBeads (Miltenyi Biotec). Ninety-six-well nitrocellulose-base plates (MultiScreen PI; Millipore) were coated overnight with an anti-murine IFN- $\gamma \mathrm{mAb}$ (Mab AN 18, $10 \mu \mathrm{g} / \mathrm{ml}$; Mabtech). The plates were washed 6 times with PBS and blocked with RPMI-1640 containing 10\% FBS at $37^{\circ} \mathrm{C}$ for 2 hours. The splenocytes or $\mathrm{CD} 8^{+}$cells, followed by irradiated, peptide-pulsed BM-derived DCs, were seeded into wells and incubated for 20 hours at $37^{\circ} \mathrm{C}$ in $5 \% \mathrm{CO}_{2}$. The cells were then removed by 6 washes with PBS $0.5 \%$ Tween-20 (Sigma-Aldrich). Biotinylated anti-mouse IFN- $\gamma$ antibody (Mab R4-6A2 biotin; Mabtech), diluted in PBS containing 0.5\% FBS to $1 \mu \mathrm{g} / \mathrm{ml}$, was added, and the mixture incubated at $25^{\circ} \mathrm{C}$ for 2 hours. The avidin/biotinylated enzyme complex (ABC; Vector Laboratories) was added for an additional hour. Cytokine-secreting cells were detected after a 4 minute reaction with AEC (3-amino-9-ethylcarbazole; Sigma-Aldrich).
The results were evaluated by ZellNet Consulting Inc. with an automated ELISPOT reader system (Zeiss), using KS ELISPOT 4.3 software.

Tetramer staining. H2-Kb/TRP2 tetramer assays were used to detect TRP2specific $\mathrm{CD}^{+} \mathrm{T}$ cells. TRP2 tetramers were synthesized at the Baylor College of Medicine Tetramer Core Facility. Splenocytes from immunized mice were double stained with anti-CD8 $\alpha$-FITC and H2-K $/$ TRP2-PE tetramers on different days after DC immunization. Tetramer staining was done at $4^{\circ} \mathrm{C}$, for 1 hour with $1 \mu \mathrm{g}$ of anti-CD8 $\alpha$ and a 1:100 dilution of TRP 2 tetramers per $10^{6}$ cells, according to the manufacturer's instructions.

Intracellular cytokine staining. Splenocytes were harvested from immunized mice and cultured with TRP2 peptide for 8 hours at $37^{\circ} \mathrm{C}$. For the final 6 hours of culture, GolgiPlug (BD Biosciences - Pharmingen) was added to the supernatant. After surface staining with anti-CD8, cells were permeabilized and stained for intracellular IFN- $\gamma$, with anti-IFN- $\gamma(\mathrm{BD})$ as we have previously described (51).

Western blotting. SOCS1-siRNA DCs or GFP-siRNA DCs were continuously stimulated with LPS (Sigma-Aldrich) and plate-immobilized anti-CD40 $\mathrm{mAb}$ (BD Biosciences - Pharmingen). Twenty-four, 48, or 72 hours later, the cells were harvested and subjected to SDS-PAGE (20). Following transfer to Hybond-P membrane (Amersham Biosciences), the samples were analyzed by Western blotting with monoclonal anti-pStat4 (Santa Cruz Biotechnology Inc.) or polyclonal anti-Stat4 (Santa Cruz Biotechnology Inc.) antibodies, followed by detection with ECL-Plus reagent (Amersham Biosciences).

DC transfer and tumor mouse study. BM-derived DCs (day 4-5 of BM culture) were transduced with LV-SOCS1-siRNA or LV-GFP-siRNA at an MOI of 5 or Ad-IL-12 vector at an MOI of 300 or 1,000 (20). DCs were then pulsed with TRP2 peptides for 20 hours, washed with PBS 3 times, stimulated with LPS (100 ng/ml; Sigma-Aldrich) or TNF- $\alpha$ ( $50 \mathrm{ng} / \mathrm{ml}$; R\&D Systems) for 24 hours, washed with PBS 3 times, and then injected into C57BL/ 6 mice via a rear foot pad. In the therapeutic model, B16 tumor cells $\left(2.5 \times 10^{5}\right)$ were injected s.c. into the right flank of syngeneic mice to establish a tumor model. On day 3 after tumor inoculation, the mice were randomly divided into groups and injected with antigen-pulsed, matured, transduced DCs $\left(1.5 \times 10^{6}\right)$ or PBS control. In some mice, TLR agonist (LPS [30 $\mu \mathrm{g} / \mathrm{mouse}$; Sigma-Aldrich], poly[I:C] [50 $\mu \mathrm{g} /$ mouse; InvivoGen], peptidoglycan [25 $\mu \mathrm{g} /$ mouse; InvivoGen], imiquimod [50 $\mu \mathrm{g} /$ mouse; InvivoGen]) or IL-12 $(1 \mu \mathrm{g} /$ mouse; PeproTech) was administered i.p. after DC vaccination on the days indicated in the figures. Tumor volumes were measured every 2-4 days with an electronic caliper until the experiment was completed.

Statistics. For statistical analysis, we used 2-tailed Student's $t$ test, and a $95 \%$ confidence limit was taken to be significant, defined as $P<0.05$. Results are presented as mean \pm SEM.

\section{Acknowledgments}

We thank Lisa Rollins, Wenhong Ren, Natasha Lapteva, Andrew Sharabi, Jiali Sun, and Lei Shen for technical assistance and valuable suggestions. This work was supported by grants from the NIH (R01CA90427, R0148480, and R01AI48711), the Leukemia and Lymphoma Society SCOR, and the US Army Prostate Cancer Research Program (to X.F. Huang). K. Evel-Kabler and M. Aldrich were supported by a predoctoral and postdoctoral NIH training grant (T32-AI07495).

Received for publication July 5, 2005, and accepted in revised form September 18, 2005.

Address correspondence to: Si-Yi Chen, Center for Cell and Gene Therapy, Alkek Building N1004, Baylor College of Medicine, One Baylor Plaza, Houston, Texas 77030, USA. Phone: (713) 798-1236; Fax: (713) 798-1083; E-mail: sychen@bcm.tmc.edu. 
1. Akira, S., and Takeda, K. 2004. Toll-like receptor signalling. Nat. Rev. Immunol. 4:499-511.

2. Beutler, B., and Rietschel, E.T. 2003. Innate immune sensing and its roots: the story of endotoxin. Nat. Rev. Immunol. 3:169-176.

3. Janeway, C.A., Jr., and Medzhitov, R. 2002. Innate immune recognition. Annu. Rev. Immunol. 20:197-216.

4. Banchereau, J., and Steinman, R.M. 1998. Dendritic cells and the control of immunity. Nature. 392:245-252.

5. Steinman, R.M., Hawiger, D., and Nussenzweig, M.C. 2003. Tolerogenic dendritic cells. Annu. Rev. Immunol. 21:685-711

6. Gilboa, E. 2004. The promise of cancer vaccines. Nat. Rev. Cancer. 4:401-411.

7. Dustin, M.L., and Cooper, J.A. 2000. The immunological synapse and the actin cytoskeleton: molecular hardware for $\mathrm{T}$ cell signaling. Nat. Immunol. 1:23-29.

8. Kaech, S.M., and Ahmed, R. 2001. Memory CD8+ T cell differentiation: initial antigen encounter triggers a developmental program in naive cells. Nat. Immunol. 2:415-422.

9. van Stipdonk, M.J., Lemmens, E.E., and Schoen berger, S.P. 2001. Naive CTLs require a single brief period of antigenic stimulation for clonal expansion and differentiation. Nat. Immunol. 2:423-429.

10. Lanzavecchia, A., and Sallusto, F. 2001. Antigen decoding by $\mathrm{T}$ lymphocytes: from synapses to fate determination. Nat. Immunol. 2:487-492.

11. Gett, A.V., Sallusto, F., Lanzavecchia, A., and Geginat, J. 2003. T cell fitness determined by signal strength. Nat. Immunol. 4:355-360.

12. Curtsinger, J.M., Lins, D.C., and Mescher, M.F. 2003. Signal 3 determines tolerance versus full activation of naive CD8 T cells: dissociating proliferation and development of effector function. J. Exp. Med. 197:1141-1151.

13. Valenzuela, J., Schmidt, C., and Mescher, M. 2002. The roles of IL-12 in providing a third signal for clonal expansion of naive CD8 T cells. J. Immunol. 169:6842-6849.

14. Nakagawa, R., et al. 2002. SOCS-1 participates in negative regulation of LPS responses. Immunity. 17:677-687.

15. Kinjyo, I., et al. 2002. SOCS1/JAB is a negative regulator of LPS-induced macrophage activation. Immunity. 17:583-591.

16. Hanada, T., et al. 2003. Suppressor of cytokine signaling- 1 is essential for suppressing dendritic cell activation and systemic autoimmunity. Immunity. 19:437-450

17. Kubo, M., Hanada, T., and Yoshimura, A. 2003 Suppressors of cytokine signaling and immunity. Nat. Immunol. 4:1169-1176.

18. Marine, J.C., et al. 1999. SOCS1 deficiency causes a lymphocyte-dependent perinatal lethality. Cell. 98:609-616.

19. Alexander, W.S., et al. 1999. SOCS1 is a critical inhibitor of interferon gamma signaling and prevents the potentially fatal neonatal actions of this cytokine. Cell. 98:597-608.

20. Shen, L., Evel-Kabler, K., Strube, R., and Chen, S.Y. 2004. Silencing of SOCS1 enhances antigen presentation by dendritic cells and antigen-specific anti- tumor immunity. Nat. Biotechnol. 22:1546-1553.

21. Hanada, T., et al. 2005. Induction of hyper Th1 cell-type immune responses by dendritic cells lacking the suppressor of cytokine signaling-1 gene. J. Immunol. 174:4325-4332.

22. Simpson, A.J., Caballero, O.L., Jungbluth, A., Chen, Y.T., and Old, L.J. 2005. Cancer/testis antigens, gametogenesis and cancer. Nat. Rev. Cancer. 5:615-625.

23. Timmerman, J.M., and Levy, R. 1999. Dendritic cell vaccines for cancer immunotherapy. Annu. Rev. Med. 50:507-529.

24. Fong, L., and Engleman, E.G. 2000. Dendritic cells in cancer immunotherapy. Annu. Rev. Immunol. 18:245-273.

25. Rosenberg, S.A., Yang, J.C., and Restifo, N.P. 2004. Cancer immunotherapy: moving beyond current vaccines. Nat. Med. 10:909-915.

26. Sakaguchi, S. 2004. Naturally arising CD4+ regulatory t cells for immunologic self-tolerance and negative control of immune responses. Annu. Rev. Immunol. 22:531-562.

27. Yang, L., and Carbone, D.P. 2004. Tumor-host immune interactions and dendritic cell dysfunction. Adv. Cancer Res. 92:13-27.

28. van Elsas, A., et al. 2001. Elucidating the autoimmune and antitumor effector mechanisms of a treatment based on cytotoxic $\mathrm{T}$ lymphocyte antigen-4 blockade in combination with a B16 melanoma vaccine: comparison of prophylaxis and therapy. J. Exp. Med. 194:481-489.

29. Yang, Y., Huang, C.T., Huang, X., and Pardoll, D.M. 2004. Persistent Toll-like receptor signals are required for reversal of regulatory $\mathrm{T}$ cell-mediated CD8 tolerance. Nat. Immunol. 5:508-515.

30. Gautier, G., et al. 2005. A type I interferon autocrine-paracrine loop is involved in Toll-like receptor-induced interleukin-12p70 secretion by dendritic cells. J. Exp. Med. 201:1435-1446.

31. Langenkamp, A., Messi, M., Lanzavecchia, A., and Sallusto, F. 2000. Kinetics of dendritic cell activation: impact on priming of TH1, TH2 and nonpolarized T cells. Nat. Immunol. 1:311-316.

32. Gingras, S., Parganas, E., de Pauw, A., Ihle, J.N., and Murray, P.J. 2004. Re-examination of the role of suppressor of cytokine signaling 1 (SOCS1) in the regulation of Toll-like receptor signaling. J. Biol. Chem. 279:54702-54707.

33. Chong, M.M., Metcalf, D., Jamieson, E., Alexander, W.S., and Kay, T.W. 2005. Suppressor of cytokine signaling- 1 in T cells and macrophages is critical for preventing lethal inflammation. Blood. 106:1668-1675.

34. Catlett, I.M., and Hedrick, S.M. 2005. Suppressor of cytokine signaling 1 is required for the differentiation of CD4+ T cells. Nat. Immunol. 6:715-721.

35. D'Andrea, A., Ma, X., Aste-Amezaga, M., Paganin, C., and Trinchieri, G. 1995. Stimulatory and inhibitory effects of interleukin (IL)- 4 and IL-13 on the production of cytokines by human peripheral blood mononuclear cells: priming for IL-12 and tumor necrosis factor alpha production. J. Exp. Med. 181:537-546.

36. Trinchieri, G. 2003. Interleukin-12 and the regulation of innate resistance and adaptive immunity. Nat. Rev. Immunol. 3:133-146.
37. Barnes, B.J., et al. 2004. Global and distinct targets of IRF-5 and IRF-7 during innate response to viral infection. J. Biol. Chem. 279:45194-45207.

38. Ohteki, T., et al. 1999. Interleukin 12-dependent interferon gamma production by CD8alpha+ lymphoid dendritic cells. J. Exp. Med. 189:1981-1986.

39. Garg, S., et al. 2003. Genetic tagging shows increased frequency and longevity of antigen-presenting, skin-derived dendritic cells in vivo. Nat. Immunol. 4:907-912.

40. Machen, J., et al. 2004. Antisense oligonucleotides down-regulating costimulation confer diabetespreventive properties to nonobese diabetic mouse dendritic cells. J. Immunol. 173:4331-4341.

41. van Elsas, A., Hurwitz, A.A., and Allison, J.P. 1999. Combination immunotherapy of B16 melanoma using anti-cytotoxic $\mathrm{T}$ lymphocyte-associated antigen 4 (CTLA-4) and granulocyte/macrophage colony-stimulating factor (GM-CSF)-producing vaccines induces rejection of subcutaneous and metastatic tumors accompanied by autoimmune depigmentation. J. Exp. Med. 190:355-366.

42. Waldner, H., Collins, M., and Kuchroo, V.K. 2004. Activation of antigen-presenting cells by microbial products breaks self tolerance and induces autoimmune disease. J. Clin. Invest. 113:990-997. doi:10.1172/JCI200419388.

43. Duan, L., Reddi, A.L., Ghosh, A., Dimri, M., and Band, H. 2004. The Cbl family and other ubiquitin ligases: destructive forces in control of antigen receptor signaling. Immunity. 21:7-17.

44. Egen, J.G., Kuhns, M.S., and Allison, J.P. 2002. CTLA-4: new insights into its biological function and use in tumor immunotherapy. Nat. Immunol. 3:611-618.

45. Hodi, F.S., et al. 2003. Biologic activity of cytotoxic T lymphocyte-associated antigen 4 antibody blockade in previously vaccinated metastatic melanoma and ovarian carcinoma patients. Proc. Natl. Acad. Sci. U. S. A. 100:4712-4717.

46. Phan, G.Q., et al. 2003. Cancer regression and autoimmunity induced by cytotoxic $\mathrm{T}$ lymphocyte-associated antigen 4 blockade in patients with metastatic melanoma. Proc. Natl. Acad. Sci. U. S. A. 100:8372-8377.

47. Eyles, J.L., Metcalf, D., Grusby, M.J., Hilton, D.J., and Starr, R. 2002. Negative regulation of interleukin-12 signaling by suppressor of cytokine signaling-1. J. Biol. Chem. 277:43735-43740.

48. Banchereau, J., Pascual, V., and Palucka, A.K. 2004. Autoimmunity through cytokine-induced dendritic cell activation. Immunity. 20:539-550.

49. Grohmann, U., et al. 1998. IL-12 acts directly on DC to promote nuclear localization of NF-kappaB and primes DC for IL-12 production. Immunity. 9:315-323.

50. You, Z., et al. 2000. Induction of vigorous helper and cytotoxic $\mathrm{T}$ cell as well as B cell responses by DCs expressing a modified antigen targeting receptor-mediated internalization pathway. J. Immunol. 165:4581-4592.

51. Hauser, H., Shen, L., Gu, Q.L., Krueger, S., and Chen, S.-Y. 2004. Secretory heat-shock protein as a dendritic cell-targeting molecule: a new strategy to enhance the potency of genetic vaccines. Gene Ther. 11:924-932. 